\title{
Conforming polygonal finite elements
}

\author{
N. Sukumar*, ${ }^{\dagger}$ and A. Tabarraei \\ Department of Civil and Environmental Engineering, University of California, Davis, CA 95616, U.S.A.
}

\begin{abstract}
SUMMARY
In this paper, conforming finite elements on polygon meshes are developed. Polygonal finite elements provide greater flexibility in mesh generation and are better-suited for applications in solid mechanics which involve a significant change in the topology of the material domain. In this study, recent advances in meshfree approximations, computational geometry, and computer graphics are used to construct different trial and test approximations on polygonal elements. A particular and notable contribution is the use of meshfree (natural-neighbour, $\mathrm{nn}$ ) basis functions on a canonical element combined with an affine map to construct conforming approximations on convex polygons. This numerical formulation enables the construction of conforming approximation on $n$-gons $(n \geqslant 3)$, and hence extends the potential applications of finite elements to convex polygons of arbitrary order. Numerical experiments on second-order elliptic boundary-value problems are presented to demonstrate the accuracy and convergence of the proposed method. Copyright (c) 2004 John Wiley \& Sons, Ltd.
\end{abstract}

KEY WORDS: meshfree methods; natural neighbour interpolants; natural element method; Laplace interpolant; Wachspress basis functions; mean value co-ordinates

\section{INTRODUCTION}

The finite element method is the ubiquitous choice to solve boundary-value problems in solid mechanics. In two-dimensions, the constant strain three-node triangular element and the bilinear four-node quadrilateral element [1,2] are widely used in research as well as in industrial practice. The development of finite elements on irregular polygons has not been explored to any significant depth so far. Wachspress [3] proposed rational basis functions on polygonal elements, which has only received limited attention so far [4-8]. The advantages and potential benefits of using polygonal finite elements in computation are striking: greater flexibility (single algorithm suffices) in the meshing of arbitrary geometries such as those that arise in biomechanics $[9,10]$; better accuracy in the numerical solution (higher-order approximation) over that obtainable using triangular and quadrilateral meshes on a given nodal grid; useful as a transition element

\footnotetext{
${ }^{*}$ Correspondence to: N. Sukumar, Department of Civil and Environmental Engineering, University of California, One Shields Avenue, Davis, CA 95616, U.S.A.

†E-mail: nsukumar@ucdavis.edu

Contract/grant sponsor: National Science Foundation; contract/grant number: CMS-0352654
}

Received 23 January 2004

Revised 26 April 2004

Copyright (c) 2004 John Wiley \& Sons, Ltd.

Accepted 21 May 2004 
in finite element meshes [11,12]; suitable in material design [13] and in the modelling of polycrystalline materials [14]; and will have less sensitivity to lock (unlike lower-order triangular and quadrilateral elements which tend to be stiff) under volume-preserving deformation states which arise in incompressible elasticity as well as in von Mises plasticity. To this end, we explore the construction of robust and accurate finite element methods that are based on polygonal elements.

In this paper, we integrate some of the recent advances in meshfree methods, computational geometry, and computer graphics to construct conforming trial and test approximations on polygonal meshes. In Section 2, the data interpolation problem on polygonal domains is described, and in Section 2.1, natural neighbour shape functions are used to construct conforming approximations on polygons. Barycentric co-ordinates (Wachspress basis functions and mean value co-ordinates) are discussed in Section 2.2. The Galerkin formulation and implementation of the polygonal FEM is presented in Section 3. In Section 4, numerical examples for the patch test and convergence studies on a Poisson problem are presented to demonstrate the accuracy of the proposed method, and we close with some concluding remarks in Section 5.

\section{CONFORMING INTERPOLANTS ON POLYGONS}

Consider a bounded domain $\Omega$ in two dimensions that is described by a set $\mathbf{N}$ of $M$ scattered nodes: $\mathbf{N}=\left\{p_{1}, p_{2}, \ldots, p_{M}\right\}$. The Voronoi diagram [15] $\mathscr{V}(\mathbf{N})$ of the set $\mathbf{N}$ is a sub-division of the domain into regions $V\left(p_{i}\right)$, such that any point in $V\left(p_{i}\right)$ is closer to node $p_{i}$ than to any other node $p_{j} \in N(j \neq i)$ (Figure $1(\mathrm{a})$ ).

The region $V\left(p_{i}\right)$ is the first-order Voronoi cell for a node $p_{i}$ within the convex hull; $V\left(p_{i}\right)$ is a convex polygon (polyhedron) in $\mathbf{R}^{2}\left(\mathbf{R}^{3}\right)$. The dual of the Voronoi diagram is the Delaunay tessellation, which is constructed by connecting nodes that have a common Voronoi edge. In Figure 1(a), the Voronoi diagram and the Delaunay triangulation are shown for a nodal set consisting of seven nodes $(M=7)$.

Let us introduce a point $p$ with co-ordinate $\mathbf{x} \equiv\left(x_{1}, x_{2}\right)$ within the convex hull of the set $\mathbf{N}$ (Figure 1(a)). The problem of defining a suitable interpolant in $\Omega$ for every point $p$ is

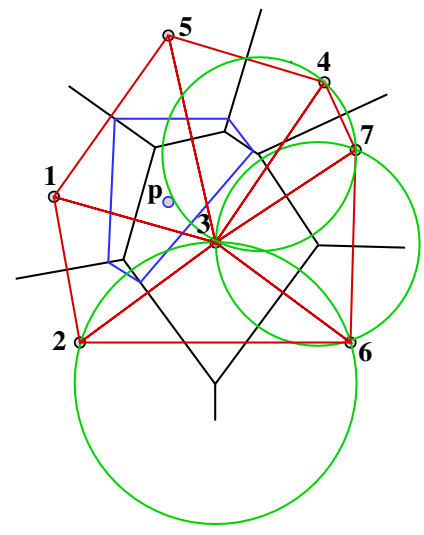

(a)

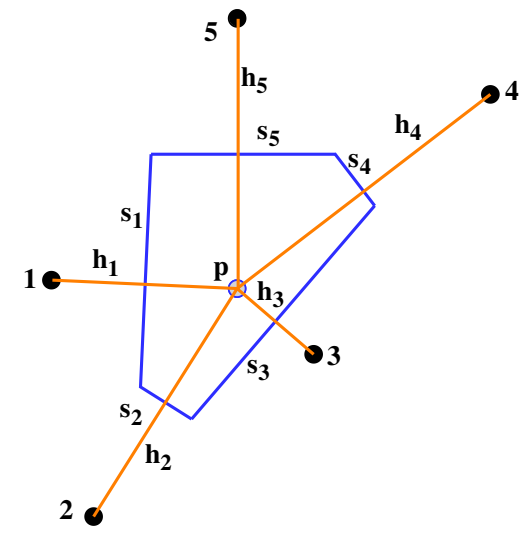

(b)

Figure 1. Natural neighbours: (a) Delaunay circumcircles; and (b) Laplace shape function. 
of interest in various applications such as partial differential equations (PDEs), scattered data approximation, computer graphics, and geometric modelling. We now focus our attention on the construction of suitable interpolating schemes for the solution of PDEs via a Bubnov-Galerkin procedure - a weak or variational statement of the governing partial differential equation is used, and the trial and test functions are represented via the same basis (shape) functions. For a scalar-valued function $u(\mathbf{x}): \Omega \rightarrow \mathbf{R}$, a general form of an interpolation scheme is:

$$
u^{h}(\mathbf{x})=\sum_{i=1}^{n} \phi_{i}(\mathbf{x}) u_{i}
$$

where $u_{i}(i=1,2, \ldots, n)$ are the unknowns at the $n$ neighbours of point $p$, and $\phi_{i}(\mathbf{x})$ is the shape function for node $i$.

From the viewpoint of a conforming Galerkin approximation, the following are some of the desirable properties of shape functions (barycentric co-ordinates when $p_{i}$ are the vertices of a polygonal or polyhedral domain $\Omega$ ) and of the resulting interpolant:

1. Non-negative, interpolate, and form a partition of unity:

$$
\begin{aligned}
0 \leqslant \phi_{i}(\mathbf{x}) & \leqslant 1, \quad i=1,2, \ldots, n \\
\phi_{i}\left(\mathbf{x}_{j}\right) & =\delta_{i j} \\
\sum_{i=1}^{n} \phi_{i}(\mathbf{x}) & =1
\end{aligned}
$$

where $\delta_{i j}$ is the Kronecker-delta. Equation (2a) in conjunction with Equation (2c) ensures that the interpolated result is bounded between the minimum and maximum of the nodal values: $\min _{i} u_{i} \leqslant u^{h}(\mathbf{x}) \leqslant \max _{i} u_{i}$. This is a statement of the discrete maximum principle, which is of relevance in the numerical solution of the diffusion equation. In $2 \mathrm{D}$, three-node (Delaunay) triangular finite elements satisfy the discrete maximum principle, but bilinear rectangles can violate this requirement [16]. The Kronecker-delta property in Equation (2b) indicates that the interpolated result at a node is identical to the nodal data: $u^{h}\left(\mathbf{x}_{i}\right)=u_{i}$, which is of particular relevance in the imposition of essential boundary conditions. A constant field (rigid-body motion in mechanics) can be exactly represented if Equation (2c) holds.

2. Linear precision or completeness [1]:

$$
\sum_{I=1}^{n} \phi_{i}(\boldsymbol{\xi}) \mathbf{x}_{i}=\mathbf{x}
$$

which indicates that the shape functions can exactly reproduce a linear function. In general, $k$ th-order finite elements in one-dimension satisfy $k$ th-order completeness. For secondorder PDEs, which typically arise in solid mechanics applications, constant and linear precision are sufficient conditions for convergence [17]. The above equation also defines an affine mapping (convex combination), and this fact is used to perform isoparametric mapping from a canonical (reference) element to the physical element in finite element methods [1]. 
3. On the boundary of the domain $\Omega$, the interpolant must be precisely linear, i.e. interior shape functions should not contribute if $p$ lies on the convex hull and in addition only the two adjacent nodes to $p$ must have non-zero values so that a linear interpolant is realized:

$$
u^{h}(t)=t u_{1}+(1-t) u_{2}, \quad \mathbf{x}=t \mathbf{x}_{1}+(1-t) \mathbf{x}_{2}, \quad \mathbf{x} \in \partial \Omega, \quad t \in[0,1]
$$

The above equation in conjunction with the Kronecker-delta property in Equation (2b) ensures that linear essential boundary conditions can be imposed as in finite elements.

\subsection{Natural neighbour shape functions on polygons}

The past decade has seen the emergence of meshfree approximations for the solution of PDEs in computational mechanics. With an aim to overcome the need to remesh in moving boundary and large deformation problems, there has been significant interest in these class of methods $[18,19]$. In most meshfree methods, moving least squares approximants (MLS) [20] are used to construct the trial and test functions. A striking and appealing property of MLS-approximants is the ease with which $C^{k}(\Omega)$ approximants can be constructed; however, MLS shape functions are not strictly non-negative nor do they interpolate nodal data. The latter shortcoming complicates the imposition of essential boundary conditions in a Galerkin implementation. A unified mathematical analysis of meshfree methods was recently proposed by Babuška and co-workers [21].

In this study, we use natural neighbour-based schemes, which are a promising alternative to MLS-approximations; in natural neighbour-based Galerkin methods [22-26], the Sibson co-ordinate [27] and the Laplace shape function [28-30], which are based on the notion of natural neighbours, are adopted. An overview with recent advances in this meshfree method is presented by Cueto and co-workers [31]. In Figure 1(a), the Voronoi cell for $p$ is shown. If the Voronoi cell for $p$ and that of node $p_{i}$ have a common Voronoi edge, then the node $p_{i}$ is said to be a natural neighbour of the point $p$ [27]. On using the empty circumcircle property of Delaunay triangulations, namely if $\mathscr{D} \mathscr{T}\left(p_{i}, p_{j}, p_{k}\right)$ is any Delaunay triangle of the nodal set $\mathbf{N}$, then the circumcircle of $\mathscr{D} \mathscr{T}$ contains no other nodes of $\mathbf{N}$, the natural neighbours can be readily determined. In Figure 1(a), point $p$ does not lie within the circumcircles that are shown, and hence only nodes 1-5 are neighbours of $p$. If four nodes lie on a natural neighbour circumcircle (degenerate case on a regular rectangular mesh), then for any point $p$, the four nodes at the vertices of the rectangle containing $p$ will always be natural neighbours for $p$ [24]. In essence, the natural neighbour circumcircles play a central role in defining the natural neighbours for a point $p$.

The Laplace interpolant is simpler and also computationally attractive vis-à-vis the Sibson interpolant, and in addition, it also provides a natural weighting (scaling) function for irregularly spaced nodes $[32,33]$. We now discuss the construction of the Laplace shape function, mindful that the Sibson co-ordinate is also applicable within the context of this study; a detailed description of the Sibson interpolant and its use in a Galerkin method can be found in Reference [24]. Referring to Figure 1(a), let $t_{i j}$ be the Voronoi edge that is common to $V_{i}$ and $V_{j}$, and $m\left(t_{i j}\right)$ denote the Lebesgue measure of $t_{i j}$ (length in 2D). If $p_{i}$ and $p_{j}$ do not have a common facet, then $m\left(t_{i j}\right)=0$. For the point $p$ with $n(n=5$ in Figure 1(a)) natural neighbours, the 
Laplace shape function for node $p_{i}$ can be written as [28]

$$
\phi_{i}^{l}(\mathbf{x})=\frac{\alpha_{i}(\mathbf{x})}{\sum_{j=1}^{n} \alpha_{j}(\mathbf{x})}, \quad \alpha_{j}(\mathbf{x})=\frac{s_{j}(\mathbf{x})}{h_{j}(\mathbf{x})}, \quad \mathbf{x} \in \mathbf{R}^{2}
$$

where $\alpha_{i}(\mathbf{x})$ is the Laplace weight function, $m\left(t_{i}\right) \equiv s_{i}(\mathbf{x})$ is the length of the Voronoi edge associated with $p$ and node $p_{i}$, and $h_{i}(\mathbf{x})$ is the Euclidean distance between $p$ and $p_{i}$ (Figure 1(b)).

The Laplace interpolant is particularly appealing since all the desirable properties indicated in Equations (2)-(4) are met by the Laplace shape function [25,28]. As mentioned earlier, due to the satisfaction of the above properties, essential boundary conditions within a Galerkin procedure are treated as in finite elements. In a prior study [25], the Laplace interpolant was shown to reduce to finite element interpolation for certain specific cases: in 1D, the interpolant is identical to linear finite element interpolation and in $2 \mathrm{D}$, if a point $p$ has three neighbours, barycentric co-ordinates are obtained and for the case of four natural neighbours $(n=4)$ at the vertices of a rectangle, bilinear interpolation on the rectangle is obtained. The Laplace shape function is $C^{0}(\Omega)$ : discontinuities in the derivatives arise at nodal locations as well as on the boundary of the support of the shape function [25,34]. The above commonalities between the Laplace and the finite element interpolants indicate that the finite element interpolant can be viewed as an instance of the more general Laplace interpolant. This viewpoint provides motivation for the formulation that follows.

We now present a technique to construct conforming approximations on convex polygons using natural neighbour (Laplace) shape functions. To this end, similar to three-node triangular and four-node quadrilateral finite elements, we define the shape functions on a canonical element with co-ordinate $\xi \equiv\left(\xi_{1}, \xi_{2}\right) \in \Omega_{0}$. In Figure 2, the canonical elements for a triangle, square, pentagon and hexagon are shown; note that in each case the nodes lie on the same circumcircle and hence all the nodes at the vertices of the polygon are the natural neighbours for any point in $\Omega_{0}$. In the computer program, the nodal co-ordinates of an $n$-gon are set as: $(\cos 2 \pi / n, \sin 2 \pi / n),(\cos 4 \pi / n, \sin 4 \pi / n), \ldots,(1,0)$. Since $\phi_{i}^{l}$ is piece-wise linear on the boundary $\partial \Omega_{0}$, we use the isoparametric mapping $\mathbf{x}=\mathbf{x}(\xi)=\sum_{i=1}^{n} \phi_{i}^{l} \mathbf{x}_{i}$ with $\phi_{i}^{l} \equiv \phi_{i}^{l}(\xi)$ defined in the reference co-ordinate system. Since the mapping is affine, the shape functions remain linear on the boundary of a distorted but convex polygon. This ensures that the shape functions are continuous, i.e. $\phi_{i} \in C^{0}(\Omega) \subset H^{1}(\Omega)$, and therefore a conforming approximation is realized. In Figure 3, the mapping for a hexagonal element is illustrated. Letting $\mathbf{J}_{2}=\partial \mathbf{x} / \partial \boldsymbol{\xi}$ be the Jacobian matrix of the transformation, the shape function derivatives in the physical co-ordinate system $\mathbf{x} \equiv\left(x_{1}, x_{2}\right)$ are determined via the relation: $\nabla \phi_{i}^{l}=\mathbf{J}_{2}^{-1} \nabla_{\xi} \phi_{i}^{l}$, where $\nabla_{\boldsymbol{\xi}}$ denotes the gradient with respect to the reference co-ordinates. Note that if the radius of the circumcircle, $r=\sqrt{2}$, then $\Omega_{0}$ in Figure 2(b) is the bi-unit square on which bilinear finite element shape functions are defined, whereas if $r=1 / \sqrt{2}$ with nodes located at $(0,0),(1,0)$, and $(0,1)$, then $\Omega_{0}$ is the same as the reference element for the three-node constant strain triangle. In the above two cases, the Laplace and finite element shape functions are identical [25]. Hence we note that the proposed numerical formulation enables the construction of conforming approximation on $n$-gons $(n>3)$, thereby extending the potential applications of finite elements to convex polygons of arbitrary order.

The above approach to construct conforming approximations extends to polyhedral meshes in $3 \mathrm{D}$ too. In a recent study, Idelsohn and co-workers $[35,36]$ used the Laplace shape function 

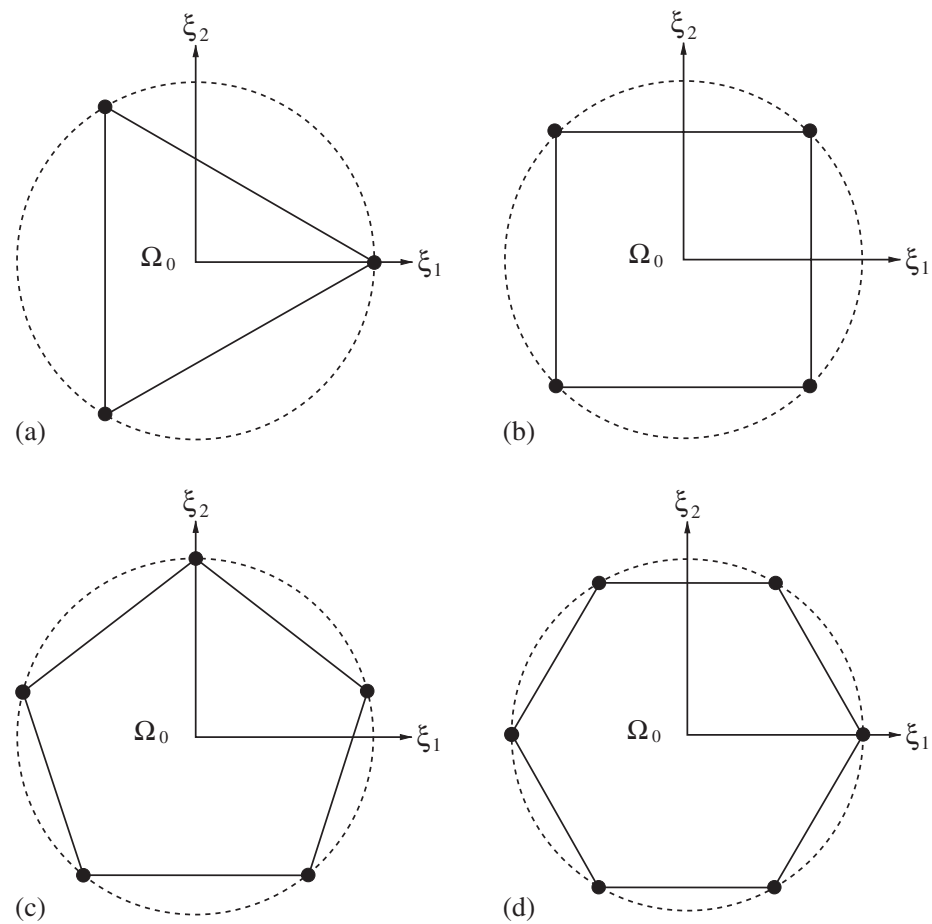

Figure 2. Canonical elements: (a) triangle; (b) square; (c) pentagon; and (d) hexagon.

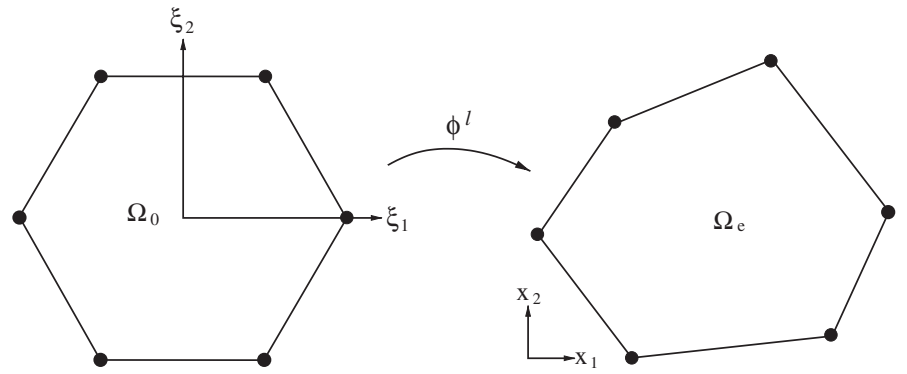

Figure 3. Isoparametric mapping.

to define a conforming interpolant on polyhedral elements in $3 \mathrm{D}$; the motivation in Reference [35] was towards the simplification of tetrahedral mesh generation. In References [35, 36], the Laplace shape functions were used on polyhedral elements in the physical space in which the nodes of the polyhedron $\left(\mathbf{x}_{i} \in \Omega\right)$ were located on the boundary of the same circumsphere (degenerate case in 3D). In the present work, no such restrictions are placed on the nodal locations, and hence the proposed method is applicable to any (convex) polygonal or polyhedral mesh. 


\subsection{Wachspress and mean value shape functions on polygons}

In this section, we present the construction of barycentric co-ordinates (Wachspress and mean value shape functions) on irregular polygons and explore their use in the formulation of polygonal finite elements. On using the principles of perspective geometry [37], Wachspress [3] proposed rational (ratio of polynomial functions) basis functions on polygonal elements, in which the algebraic equations of the edges were used to ensure nodal interpolation and linearity on the boundaries. The numerical analysis of these basis functions [4], its use to construct surface patches [5], and its generalization to convex polytopes [38] have been explored. However, its use within finite element methods has been scarce. Recently, Dasgupta and Malsch [6-8] have used symbolic computations to compute the Wachspress basis functions and also explored the construction of shape functions for concave elements. In computational solid mechanics, Rashid and Gullett [39] proposed a variable element topology finite element method (VETFEM), in which shape functions for convex and non-convex elements are constructed in the physical space $(\mathbf{x} \in \Omega)$ using a constrained minimization procedure. The VETFEM implementation falls within the class of non-conforming methods and hence as opposed to conforming methods, here consistency and the satisfaction of the patch test $[40,41]$ need to be addressed [39].

In computer graphics, surface parameterization and morphing are applications in which data interpolation schemes are of primary importance. It is also desirable in these applications that the barycentric co-ordinates on polygons satisfy, among others, the properties indicated in Equations (2)-(4). The Wachspress basis functions have re-surfaced into prominence and efforts are also underway to develop meshfree parameterization of data sets. We focus on two recent and important results that are germane to our explorations on polygonal finite elements: a generalized barycentric co-ordinate for $n$-gons [42] and mean value co-ordinates [43], which is particularly striking. In Reference [42], a simple expression is obtained for Wachspress's basis functions [3]:

$$
\phi_{i}^{w}(\mathbf{x})=\frac{w_{i}(\mathbf{x})}{\sum_{j=1}^{n} w_{j}(\mathbf{x})}, \quad w_{i}(\mathbf{x})=\frac{A\left(p_{i-1}, p_{i}, p_{i+1}\right)}{A\left(p_{i-1}, p_{i}, p\right) A\left(p_{i}, p_{i+1}, p\right)}=\frac{\cot \gamma_{i}+\cot \delta_{i}}{\left\|\mathbf{x}-\mathbf{x}_{i}\right\|^{2}}
$$

where the last expression is due to Meyer and co-workers [42]. In the above equation, $A(a, b, c)$ is the signed area of triangle $[a, b, c]$, and $\gamma_{i}$ and $\delta_{i}$ are shown in Figure 5(a). Since the numerator $\cot \gamma_{i}+\cot \delta_{i}=\sin \left(\gamma_{i}+\delta_{i}\right) /\left(\sin \gamma_{i} \sin \delta_{i}\right)$, we note that for $\phi_{i}^{w}$ to be non-negative, the polygon must be convex $\left(\gamma_{i}+\delta_{i}<\pi\right)$. The evaluation of the Wachspress basis function is carried out using elementary vector calculus operations. The angles $\delta_{i}$ and $\gamma_{i}$ are not explicitly computed (susceptible to round-off errors), and hence as suggested in Reference [42], vector cross product and vector dot product formulas are used to find the cotangents. For instance, let the co-ordinates of the vertices of triangle $\left[p_{i}, p_{i+1}, p\right]$ be $\left(a_{1}, a_{2}\right),\left(b_{1}, b_{2}\right)$, and $\left(x_{1}, x_{2}\right)$, respectively. Then,

$$
\cot \delta_{i}=\frac{\left(\mathbf{p}_{i+1}-\mathbf{p}_{i}\right) \cdot\left(\mathbf{p}-\mathbf{p}_{i}\right)}{\left|\left(\mathbf{p}_{i+1}-\mathbf{p}_{i}\right) \times\left(\mathbf{p}-\mathbf{p}_{i}\right)\right|}=\frac{\left(b_{1}-a_{1}\right)\left(x_{1}-a_{1}\right)+\left(b_{2}-a_{2}\right)\left(x_{2}-a_{2}\right)}{\left(b_{1}-a_{1}\right)\left(x_{2}-a_{2}\right)-\left(x_{1}-a_{1}\right)\left(b_{2}-a_{2}\right)} \equiv \frac{C}{S}
$$

and its derivatives are:

$$
\frac{\partial\left(\cot \delta_{i}\right)}{\partial x_{1}}=\frac{\left(b_{1}-a_{1}\right)-\cot \delta_{i}\left(a_{2}-b_{2}\right)}{S}, \quad \frac{\partial\left(\cot \delta_{i}\right)}{\partial x_{2}}=\frac{\left(b_{2}-a_{2}\right)-\cot \delta_{i}\left(b_{1}-a_{1}\right)}{S}
$$




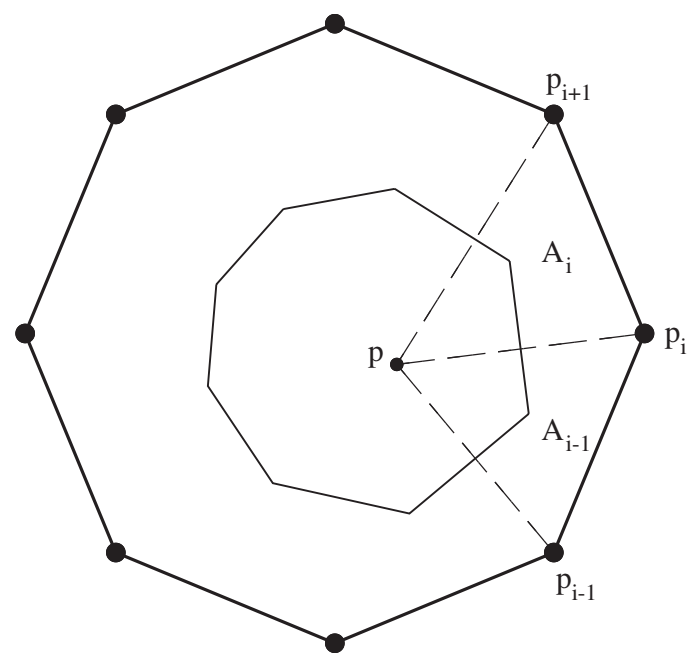

Figure 4. Equivalence of Laplace and Wachspress shape functions on a regular $n$-gon $(n=8)$.

In a similar manner, we compute $\cot \gamma_{i}$, which appears in Equation (6), and thereafter the Wachspress shape function $\phi_{i}^{w}$ and its derivatives are evaluated.

On a regular $n$-gon (Figure 2), the Wachspress shape functions are identical to Laplace shape functions; however, on arbitrary convex polygons the two shape functions are distinct. The above equivalence has been verified through numerical computations, and a proof towards the same end follows.

Proof

Consider the regular octagon shown in Figure 4, where a point $p$ is located in its interior. For matter of convenience and ease of exposition, we use Cartesian co-ordinates (rather than the local $\xi$-co-ordinates) to denote the position of $p$ and the nodes. The Voronoi polygon associated with $p$ is also illustrated. From Equations (5) and (6), we have

$$
\phi_{i}^{w}=\frac{w_{i}}{\sum_{j=1}^{n} w_{j}}, \quad \phi_{i}^{l}=\frac{\alpha_{i}}{\sum_{j=1}^{n} \alpha_{j}}
$$

and hence to show that $\phi_{i}^{w}=\phi_{i}^{l}$, it suffices if we prove that

$$
\frac{\alpha_{i}}{w_{i}}=B \quad \forall i
$$

where $B$ may depend on the co-ordinates of $p$ but is independent of the nodal co-ordinates. From Equation (6), we have

$$
w_{i}=\frac{A\left(p_{i-1}, p_{i}, p_{i+1}\right)}{A\left(p_{i-1}, p_{i}, p\right) A\left(p_{i}, p_{i+1}, p\right)}=\frac{A}{A_{i-1} A_{i}}=\frac{4 \sin ^{3} \pi / n \cos \pi / n}{A_{i-1} A_{i}}
$$

where $A \equiv A\left(p_{i-1}, p_{i}, p_{i+1}\right)$ is the area of the isosceles triangle $\left[p_{i-1}, p_{i}, p_{i+1}\right]$ with common edge length $s=2 \sin (\pi / n)$ and common angle $\pi / n$. Now, we use an expression derived in 
Reference [25] for the Laplace weight $\alpha_{i}$. On reproducing Equation (40) from Reference [25], we have

$$
2 \alpha_{i}=\frac{s_{i}}{h_{i}}=\left|\ell_{i}-r_{i}\right|
$$

where

$$
\begin{aligned}
& \ell_{i}=\frac{\left(x_{i}-x_{i-1}\right)\left(x_{i-1}-x\right)+\left(y_{i}-y_{i-1}\right)\left(y_{i-1}-y\right)}{\left(x_{i}-x\right)\left(y_{i-1}-y\right)-\left(x_{i-1}-x\right)\left(y_{i}-y\right)} \\
& r_{i}=\frac{\left(x_{i}-x_{i+1}\right)\left(x_{i+1}-x\right)+\left(y_{i}-y_{i+1}\right)\left(y_{i+1}-y\right)}{\left(x_{i}-x\right)\left(y_{i+1}-y\right)-\left(x_{i+1}-x\right)\left(y_{i}-y\right)}
\end{aligned}
$$

In the above equation, the nodes of the triangle $\left[p_{i-1}, p_{i}, p_{i+1}\right]$ are oriented in counter clockwise order (Figure 4). The factor of two appears in Equation (11a), since here $h_{i}=d\left(\mathbf{x}, \mathbf{x}_{i}\right)$, whereas in Reference [25], $h_{i}$ was defined as $d\left(\mathbf{x}, \mathbf{x}_{i}\right) / 2$. Letting $2 \alpha_{i}=\left|q_{i}\right|$, we can write

$$
\begin{aligned}
q_{i}= & \frac{\left[\left(x_{i}-x_{i-1}\right)\left(x_{i-1}-x\right)+\left(y_{i}-y_{i-1}\right)\left(y_{i-1}-y\right)\right]\left[\left(x_{i}-x\right)\left(y_{i+1}-y\right)-\left(x_{i+1}-x\right)\left(y_{i}-y\right)\right]}{4 A_{i-1} A_{i}} \\
& -\frac{\left[\left(x_{i}-x_{i+1}\right)\left(x_{i+1}-x\right)+\left(y_{i}-y_{i+1}\right)\left(y_{i+1}-y\right)\right]\left[\left(x_{i}-x\right)\left(y_{i-1}-y\right)-\left(x_{i-1}-x\right)\left(y_{i}-y\right)\right]}{4 A_{i-1} A_{i}}
\end{aligned}
$$

We use polar co-ordinates (unit radius) to write $(x, y) \equiv(\cos \theta, \sin \theta)$ for $p$ and $\left(x_{m}, y_{m}\right) \equiv$ $(\cos 2 \pi m / n, \sin 2 \pi m / n)$ for the nodal co-ordinates. On using symbolic computations to reduce the numerator in the above equation, we arrive at the following simplified expression:

$$
\alpha_{i}=\frac{2\left(1-x^{2}-y^{2}\right) \sin ^{3} \pi / n \cos \pi / n}{A_{i-1} A_{i}}
$$

and hence on using Equation (10), we have

$$
\frac{\alpha_{i}}{w_{i}}=\frac{1-x^{2}-y^{2}}{2} \equiv B
$$

where $B$ is independent of the nodal co-ordinates, as required.

The extension of barycentric co-ordinates for non-convex polygons, while retaining the properties in Equations (2)-(4), is non-trivial. As indicated earlier, if we apply Equation (6) for non-convex polygons, $\phi_{i}^{w}<0$ is obtained for a vertex node whose edges are the cause for the non-convexity. In addition, the construction of an affine mapping from a non-convex polygon to a convex polygon must ensure that fold-over does not occur. An advance in this direction was recently obtained in Reference [44], where approximations to harmonic maps over triangulations was studied so that injectivity (one-to-one) of the map is preserved. It was concluded that $\phi_{\mathscr{T}}$ must be a convex combination map, i.e. every interior vertex of $\mathscr{T}$ can be represent as a weighted sum (with positive weights) of its neighbours on the boundary $\partial \mathscr{T}$. If $\phi_{\mathscr{T}}$ maps the boundary $\mathscr{T}$ homeomorphically to the boundary of a convex region, then $\phi_{\mathscr{T}}$ is one-to-one; 

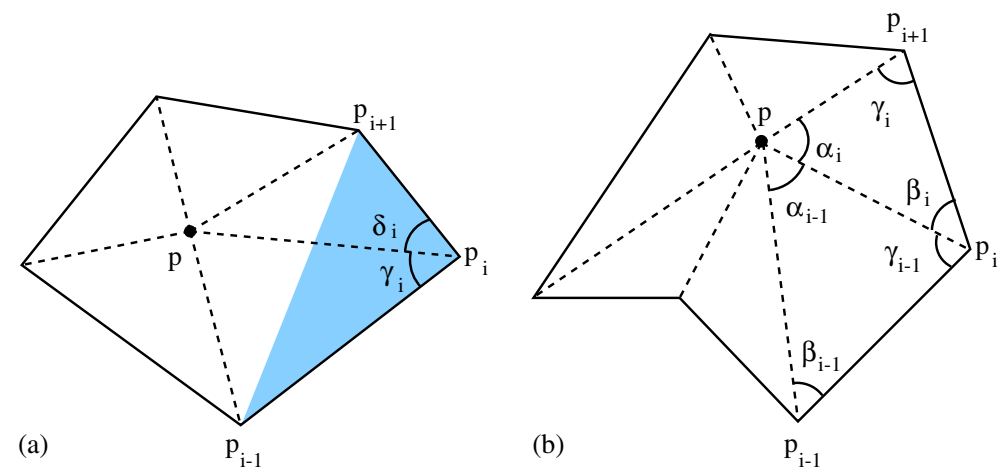

Figure 5. Barycentric co-ordinates: (a) Wachspress's basis function [42]; and (b) mean value co-ordinates [43].

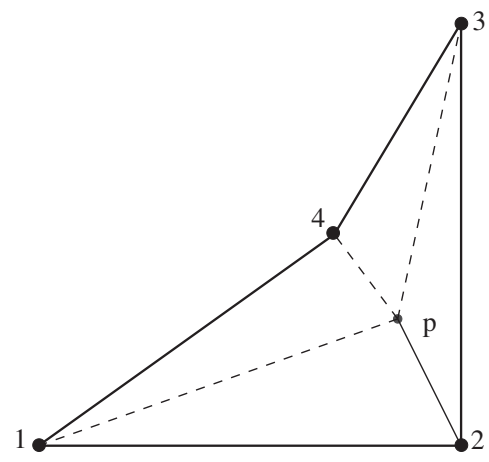

Figure 6. Mean value co-ordinates at $p$ (non-convex polygon).

details are provided in Reference [44]. Floater [43] used the mean value theorem for harmonic functions to develop non-negative weights (barycentric co-ordinate) on polygons. The linearly precise mean value co-ordinate is [43]

$$
\phi_{i}^{m}(\mathbf{x})=\frac{w_{i}(\mathbf{x})}{\sum_{j=1}^{n} w_{j}(\mathbf{x})}, \quad w_{i}(\mathbf{x})=\frac{\tan \left(\alpha_{i-1} / 2\right)+\tan \left(\alpha_{i} / 2\right)}{\left\|\mathbf{x}-\mathbf{x}_{i}\right\|}
$$

where the angle $\alpha_{i}$ is shown in Figure 5(b). We first compute the cotangent of the whole-angles (e.g. $\left.t \equiv \cot \alpha_{i}\right)$ using the formulas presented in Equation (7). Letting $w \equiv \tan \left(\alpha_{i} / 2\right)$, we have the identity $t=\left(1-w^{2}\right) / 2 w$ which is a quadratic equation in the unknown $w$. Once $t$ is evaluated, we solve the quadratic equation to find $w$ (positive root) and then its derivatives are evaluated towards the computation of $\phi_{i}^{m}$ and its derivatives. In Figure 6, we show a non-convex four-node element with nodal co-ordinates $(0,0),(1,0),(1,1)$, and $(0.7,0.5)$. The mean value co-ordinates are evaluated at the point $p$ with co-ordinate $(0.85,0.3)$. The point $p$ meets the requirements stipulated in Reference [44] to ensure a convex combination map; in essence, if the domain is triangulated with $p$ as an internal point, then the resulting triangulation must not 


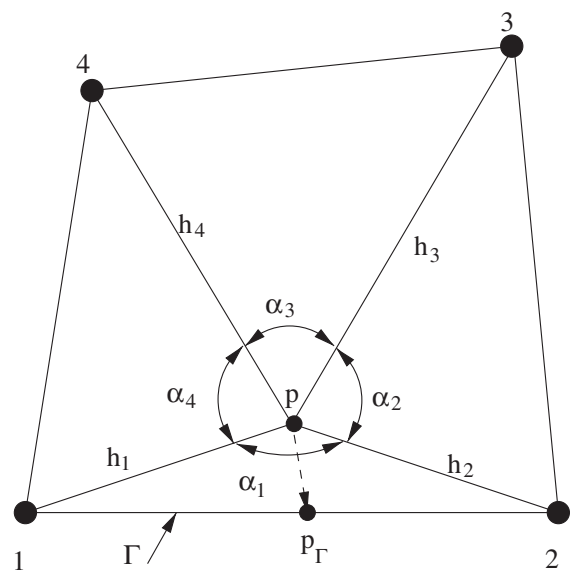

Figure 7. Linear behaviour of mean value shape functions along a boundary $\Gamma$.

contain an edge with node 2 connected directly to node 4 . Hence, it is apparent that the mean value co-ordinates are not valid for all points $p$ that lie inside the element. The linearly precise shape function values at $p$ are (4-digit precision): $\phi^{m}=\{0.0888,0.5092,0.1980,0.2040\}$. The shape functions $\phi_{i}^{m}$ are distinct from $\phi_{i}^{w}$ : the latter is identical to bilinear finite element shape functions (similar to the Laplace shape function) on a rectangle whereas the mean value shape functions are not bilinear [43].

The mean value co-ordinates are linear on the boundary of an $n$-gon, and they also satisfy the Kronecker-delta property. A proof for the above follows.

Proof

Consider the four-node quadrilateral element shown in Figure 7 , where a point $p$ is near the edge $\Gamma$. The distance between $p$ and node $i$ is denoted by $h_{i} \equiv h_{i}(\mathbf{x})$. The behaviour of the shape functions $\phi_{i}^{m}$ is studied in the limit that $p \rightarrow p_{\Gamma} \in \Gamma$. From Equation (15), we can write:

$$
w_{i}(\mathbf{x})=\frac{\tan \left(\alpha_{i-1} / 2\right)+\tan \left(\alpha_{i} / 2\right)}{h_{i}}, \quad\left(i=1-4, \quad \alpha_{0} \equiv \alpha_{4}\right)
$$

and the shape functions are

$$
\phi_{i}^{m}(p)=\frac{w_{i}(\mathbf{x})}{w_{1}(\mathbf{x})+w_{2}(\mathbf{x})+w_{3}(\mathbf{x})+w_{4}(\mathbf{x})}
$$

In the limit that $p \rightarrow p_{\Gamma}$, the angle $\alpha_{1} \rightarrow \pi$, whereas $\alpha_{2}, \alpha_{3}, \alpha_{4}<\pi$. From Equation (16), we note that in this case, $w_{1} \rightarrow \infty$ and $w_{2} \rightarrow \infty$. From the shape function definitions in Equation (17), we infer that $\phi_{3} \rightarrow 0$ and $\phi_{4} \rightarrow 0$, since $w_{3}$ and $w_{4}$ are finite. Hence the only non-zero shape functions are $\phi_{1}^{m}$ and $\phi_{2}^{m}$ :

$$
\phi_{k}^{m}\left(p_{\Gamma}\right)=\lim _{p \rightarrow p_{\Gamma}} \frac{w_{k}(\mathbf{x})}{w_{1}(\mathbf{x})+w_{2}(\mathbf{x})+w_{3}(\mathbf{x})+w_{4}(\mathbf{x})}, \quad(k=1,2)
$$


and on dividing the numerator and denominator by $w_{k}(k=1,2)$ and noting that in the limit $p \rightarrow p_{\Gamma}, w_{3} / w_{k} \rightarrow 0$ and $w_{4} / w_{k} \rightarrow 0$, we obtain

$$
\phi_{k}^{m}\left(p_{\Gamma}\right)=\lim _{p \rightarrow p_{\Gamma}} \frac{w_{k}(\mathbf{x})}{w_{1}(\mathbf{x})+w_{2}(\mathbf{x})}, \quad(k=1,2)
$$

Without loss of generality, let nodes 1 and 2 be located at $(0,0)$ and $(1,0)$, respectively. If the co-ordinate of $p_{\Gamma}$ is $\left(x_{1}, 0\right)$, then $h_{1}(\mathbf{x})=x_{1}$ and $h_{2}(\mathbf{x})=1-x_{1}$. Letting $\zeta\left(p_{\Gamma}\right)=$ $\lim _{p \rightarrow p_{\Gamma}} w_{1}(\mathbf{x}) / w_{2}(\mathbf{x})$, we can write

$$
\zeta\left(p_{\Gamma}\right)=\lim _{p \rightarrow p_{\Gamma}} \frac{\left(\tan \alpha_{1}+\tan \alpha_{4}\right) h_{2}}{\left(\tan \alpha_{1}+\tan \alpha_{2}\right) h_{1}}=\frac{h_{2}}{h_{1}}=\frac{1-x_{1}}{x_{1}}
$$

since $\lim _{p \rightarrow p_{\Gamma}} \tan \alpha_{2} / \tan \alpha_{1}=\lim _{p \rightarrow p_{\Gamma}} \tan \alpha_{4} / \tan \alpha_{1}=0$. On using the above expression for $\zeta$ in Equation (19), we obtain the desired result:

$$
\phi_{1}^{m}\left(p_{\Gamma}\right)=1-x_{1}, \quad \phi_{2}^{m}\left(p_{\Gamma}\right)=x_{1}
$$

\section{GALERKIN FORMULATION AND IMPLEMENTATION}

In this study, applications to the Poisson equation are performed to test the performance and accuracy of the polygonal finite element method. For simplicity, we focus on the following Dirichlet problem:

$$
\begin{aligned}
-\nabla^{2} u(\mathbf{x}) & =f(\mathbf{x}) \quad \text { in } \Omega \\
u & =g(\mathbf{x}) \quad \text { on } \partial \Omega
\end{aligned}
$$

The weak or variational form is

$$
\int_{\Omega} \nabla u \cdot \nabla \delta u=\int_{\Omega} f \delta u \quad \forall \delta u \in H_{0}^{1}(\Omega)
$$

where $u$ and $\delta u$, which are the trial and test approximations, respectively, are of the form shown in Equation (1), and $H_{0}^{1}(\Omega)$ is the Sobolev space of functions with square-integrable derivatives and vanishing values on $\partial \Omega$. On using the arbitrariness of nodal variations, we obtain the following system:

$$
\mathbf{K d}=\mathbf{f}, \quad \mathbf{K}_{i j}=\int_{\Omega} \nabla \phi_{i} \cdot \nabla \phi_{j} \mathrm{~d} \Omega, \quad \mathbf{f}_{i}=\int_{\Omega} f \phi_{i} \mathrm{~d} \Omega, \quad \mathbf{d}^{\mathrm{T}}=\left\{u_{1}, u_{2}, \ldots, u_{N}\right\}
$$

The domain $\Omega=\bigcup_{i} V_{i}$, where $V_{i}$ are $n$-gons. The stiffness matrix consists of the integral over $V_{i}$ of the product of shape function derivatives. The key ingredient in the proposed methodology that remains to be addressed is the evaluation of such integrals. The design and development of quadrature rules over arbitrary polygons has not yet reached a mature stage; for the difficulties associated with this endeavor and recent progress achieved in this direction, see 
Reference [39]. For the purpose of numerical integration, we present two potential solutions:

1. The first approach is to carry out numerical integration by sub-dividing the physical or the canonical element into triangles and then using well-known quadrature rules on a triangle. We point out that the partition of the $n$-gon into sub-triangles is used solely for the purpose of numerical integration, and hence within any of the sub-triangles, both the shape function $\phi_{i}$ as well as its gradient $\phi_{i, j}(i=1,2, \ldots, n, j=1,2)$ are non-zero.

2. Cubature rules on a regular hexagon are available [45], and since the canonical element is a regular $n$-gon such rules can prove to be useful. On combining elements of group theory and numerical optimization, a class of cubature rules for triangles in $\mathbf{R}^{2}$ was proposed by Wandzura and Xiao [46]. The scheme resulted in rapid convergence, had positive weights, and exhibited symmetry, much like one-dimensional Gaussian quadrature rules. The approach pursued in [46] can be extended to the design of cubature rules on arbitrary $n$-gons [47].

In this study, we use the first approach to perform numerical integration of the bilinear and linear forms that appear in the weak form. On elements with three nodes, one-point quadrature is used, whereas on four-node bilinear quadrilateral elements $2 \times 2$ Gauss-Legendre quadrature is adopted. For $n$-gons with $n>4$, we use the centroid of the element to partition it into $n$ triangles for the purpose of numerical integration. The implementation for the Wachspress and mean value shape functions is straight-forward since these are derived in the physical $\mathbf{x}$-co-ordinate itself. For the Laplace shape function, two possibilities exist: sub-division of either the (i) physical element, or the (ii) canonical element, into triangles. If the numerical integration is conducted by partitioning the physical element, then the integration of a (scalar) function $f$ over $V_{i}$ (a $n$-gon) is written as

$$
\int_{V_{i}} f \mathrm{~d} \Omega=\sum_{j=1}^{n} \int_{V_{i}^{\Delta_{j}}} f \mathrm{~d} \Omega=\sum_{j=1}^{n} \int_{0}^{1} \int_{0}^{1-\xi} f\left|\mathbf{J}_{1}^{j}\right| \mathrm{d} \xi \mathrm{d} \eta
$$

and a $n s p$-point quadrature rule on each reference right-triangle is used to compute the last integral. In the above case, for a given quadrature point, we determine $\mathbf{x}$ via: $\mathbf{x}=\sum_{i=1}^{3} N_{i} \mathbf{x}_{i}$, where $N_{i}$ are the finite element shape functions for a three-node triangle. To compute the Laplace shape function, we need the $\xi$-co-ordinates in the canonical element. To this end, we solve the isoparametric map $\mathbf{x}=\mathbf{x}(\xi)$ for $\xi$ via a Newton-Raphson procedure; a Fortran code to perform the inverse map is provided in Reference [48]. Once $\xi$ is determined, the shape functions are evaluated first in the local co-ordinates and then computed in the physical $\mathbf{x}$-co-ordinates. If the numerical integration is conducted by partitioning the canonical element, then the following transformations are performed to integrate $f$ :

$$
\int_{V_{i}} f \mathrm{~d} \Omega=\int_{\Omega_{0}} f\left|\mathbf{J}_{2}\right| \mathrm{d} \Omega=\sum_{j=1}^{n} \int_{\Omega_{0}^{\Delta_{j}}} f\left|\mathbf{J}_{2}\right| \mathrm{d} \Omega=\sum_{j=1}^{n} \int_{0}^{1} \int_{0}^{1-\xi} f\left|\mathbf{J}_{1}^{j}\right|\left|\mathbf{J}_{2}\right| \mathrm{d} \xi \mathrm{d} \eta
$$

In Equation (26), the local co-ordinate $\xi$ in the canonical element is obtained via the isoparametric map $\xi=\sum_{i=1}^{3} N_{i} \xi_{i}$, which enables the Laplace shape functions to be readily computed. This approach parallels that used in classical finite elements and hence a Newton-Raphson procedure is not required in the computations. The sequence of transformations used in this scheme are illustrated in Figure 8. 


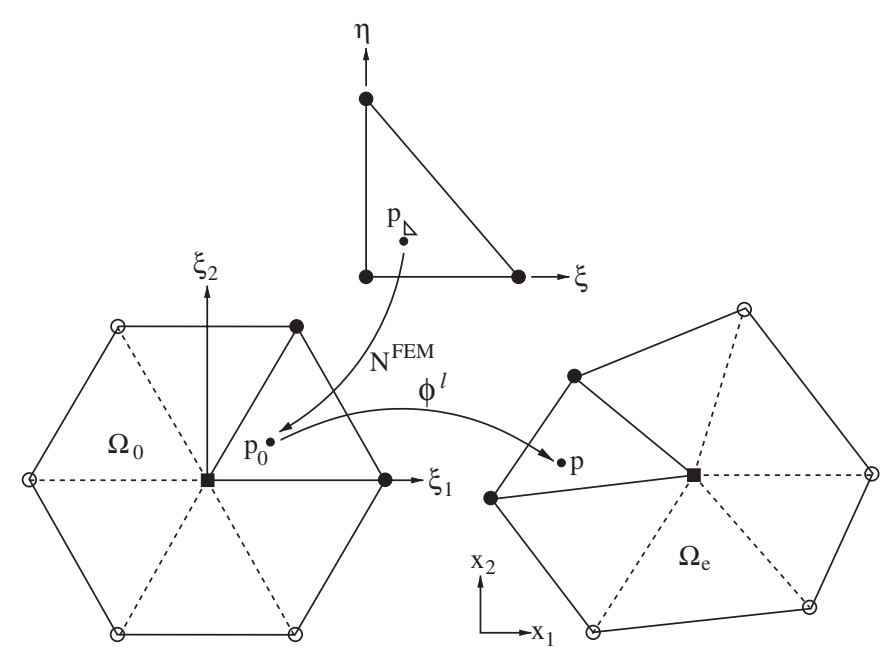

Figure 8. Numerical integration scheme based on the partition of the canonical element.

\section{NUMERICAL RESULTS}

Numerical experiments are performed using the polygonal finite element methods discussed in Section 3. We first present the shape function (Wachspress, mean value, and Laplace) plots on different $n$-gons, and also numerically verify the linearity of the mean value and Laplace shape functions on the boundary of a polygon. Then, the patch test results for the Laplace equation are studied on polygonal meshes. Lastly, a convergence study in the $L^{2}(\Omega)$ and energy norms is carried out for a Poisson problem to demonstrate the accuracy and rate of convergence of the method. In our analysis, the $L^{2}$ and energy norms are defined as

$$
\left\|u-u^{h}\right\|_{L^{2}(\Omega)}=\sqrt{\int_{\Omega}\left(u-u_{h}\right)^{2} \mathrm{~d} \Omega}, \quad\left\|u-u^{h}\right\|_{E(\Omega)}=\sqrt{\int_{\Omega}\left(u_{, i}-u_{, i}^{h}\right)\left(u_{, i}-u_{, i}^{h}\right) \mathrm{d} \Omega}
$$

where $(\cdot)_{, i}=\partial(\cdot) / \partial x_{i}$, and $u$ and $u^{h}$ are the exact and the finite element solutions, respectively.

\subsection{Polygonal shape functions}

The Laplace shape function plots for a specific node $\left(\phi_{i}\right.$ for others can be obtained via symmetry) on four different regular $n$-gons are presented in Figure 9 . These $n$-gons are the canonical elements used for the construction of the Laplace shape functions. The maximum value of $\phi$ in each case is unity (figures have been re-scaled for clarity) and the shape function is observed to be linear along the edges that includes the specific node. Consider the mesh shown in Figure 12(b), in which three elements are labeled as I, II, and III; node 2 is common to these three elements. In Figure 10, the Wachspress, mean value, and Laplace shape function plots for this node are shown. Even though the plots are visually similar, the shape function values obtained via each scheme is distinct. We observe that the shape function decays monotonically from unity at $\mathbf{x}_{2}$ to zero on the boundary of the union of the three polygons. 


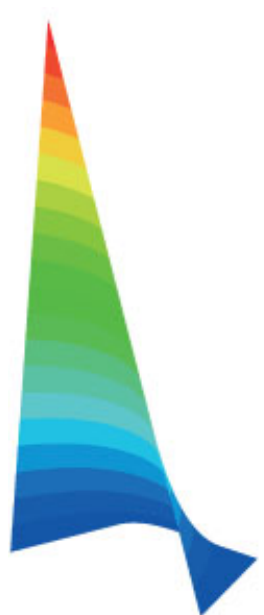

(a)

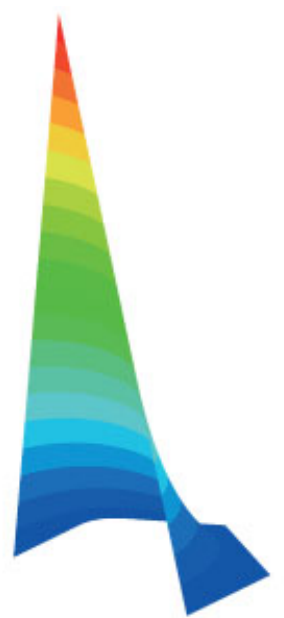

(b)

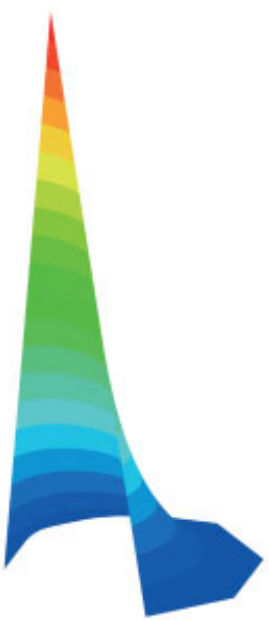

(c)

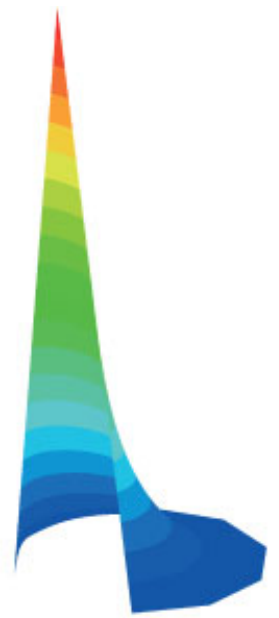

(d)

Figure 9. Laplace shape function on a regular $n$-gon: (a) $n=5$; (b) $n=6$; (c) $n=8$; and (d) $n=10$.
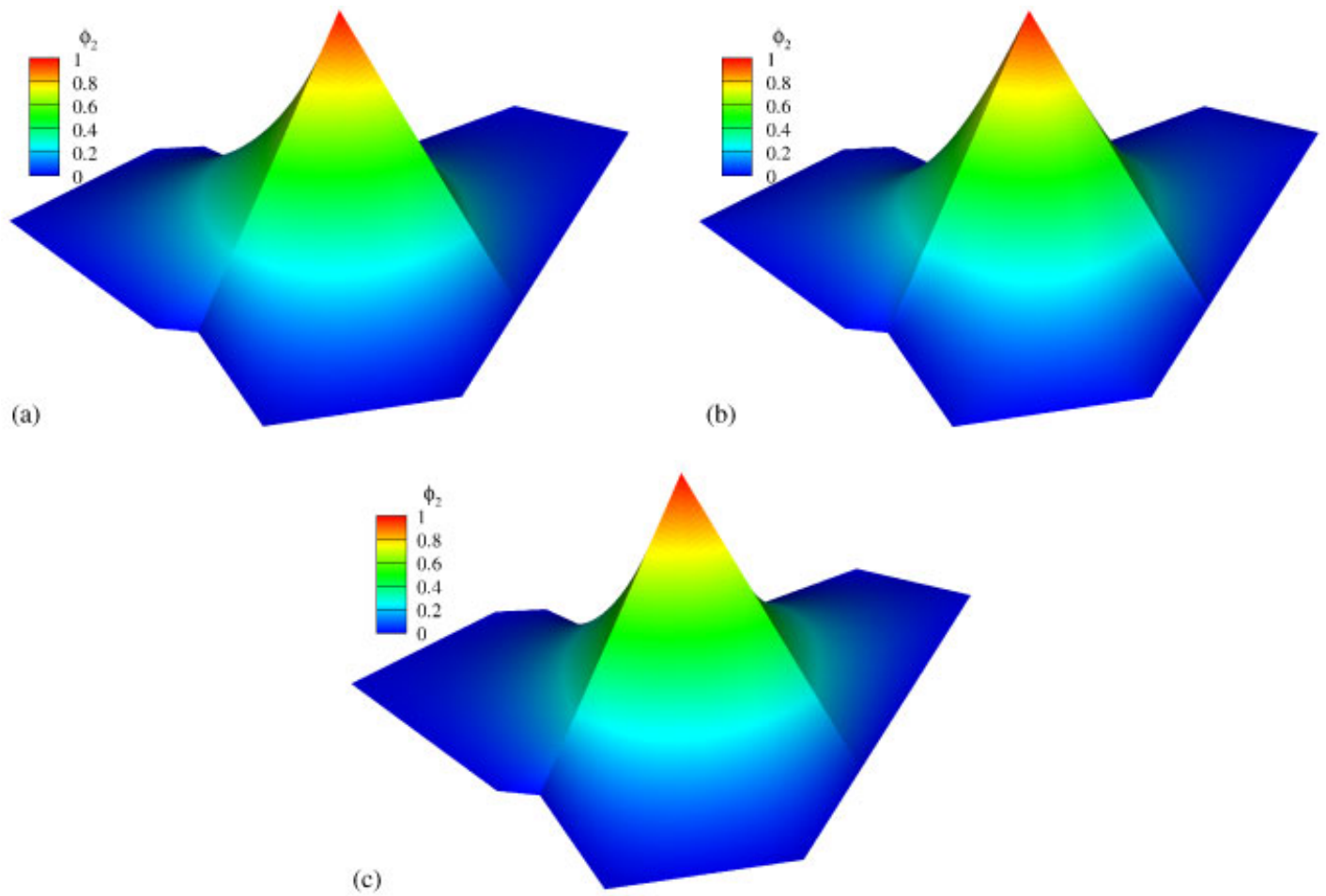

Figure 10. Shape function plots for node 2 in the mesh shown in Figure 12(b): (a) Wachspress; (b) mean-value; and (c) Laplace. 

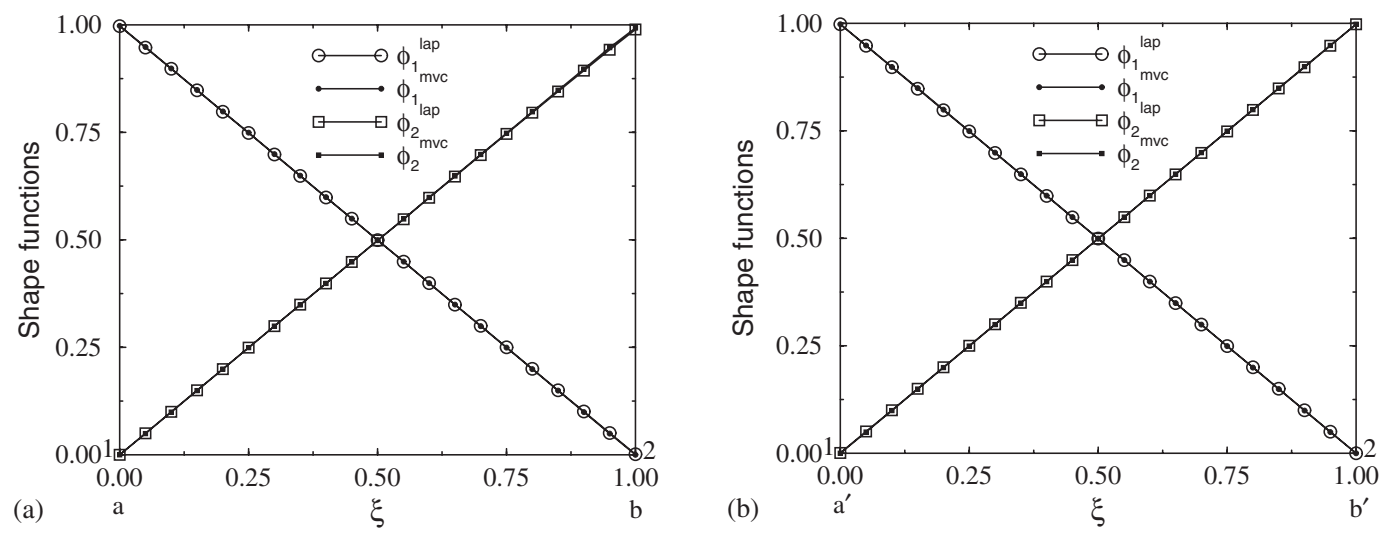

Figure 11. Mean value and Laplace shape functions near an element edge (see Figure 12(b)): (a) along $a b$; and (b) along $a^{\prime} b^{\prime}$.

In addition, the shape function is linear along all the edges where two of the polygons meet. Hence we can infer that $\phi_{i}(\mathbf{x})$ are $C^{0}(\Omega)$ functions; a precise numerical verification of this observation follows.

The nodes at the vertices of the common edge between elements I and II are labeled as 1 and 2 . To study the variation of the shape functions along the edge $1-2$, we consider lines $a b$ and $a^{\prime} b^{\prime}$ that are a distance $\varepsilon$ above and below edge 1-2 (Figure 10(b)). In Figure 11, the shape functions $\phi_{1}$ and $\phi_{2}$ are plotted along these two lines, with $\varepsilon=0.001$ used in the numerical computations. In both cases, $\phi_{1}+\phi_{2} \sim 1$ along $a b$ and $a^{\prime} b^{\prime}$, which indicates that as the edge 1-2 is approached, only these functions are non-zero, and along the edge 1-2, $\phi_{1}+\phi_{2}=1$ would be rigorously satisfied. This verifies the proof outlined in Section 2.2, in which the linear behaviour of the mean value shape functions was established. The conformity of the Wachspress shape function is well-known, and through the above computations, we have demonstrated that the mean value and Laplace shape functions also lead to conforming trial and test approximations.

\subsection{Patch test}

The patch test for the Laplacian operator is carried out: $\nabla^{2} u=0$ in $\Omega=(-1,1)^{2}$, with $u=g(\mathbf{x})=x_{1}+x_{2}$ imposed on the boundary of the bi-unit square. The exact solution is: $u(\mathbf{x})=x_{1}+x_{2}$. In the analyses, four different meshes are considered: the number of elements (nodes) are 4 (10), 10 (22), 40 (82), and 200 (402), respectively (Figure 12). For a triangular or quadrilateral element in the mesh, finite element (or equivalently Laplace) shape functions are always used. The number of quadrature points ( $n s p)$ is varied: $3,6,13$, and 25 sampling points are used, and results are presented for the two integration schemes (partitioning the physical element and the reference element) discussed in Section 3. In all cases, the norm computations are carried out by numerical integration on the physical element. The relative error in the $L^{2}$ norm and the energy norm are presented in Tables I and II, respectively. For both the integration schemes, the errors decrease with increase in the number of quadrature points $(n s p)$. 


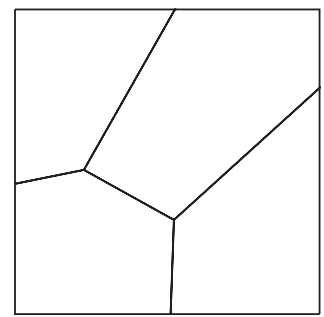

(a)

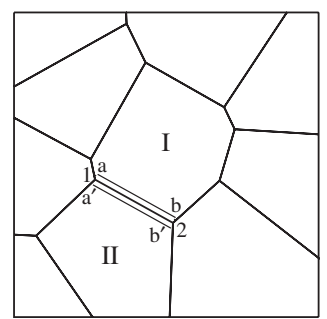

(b)

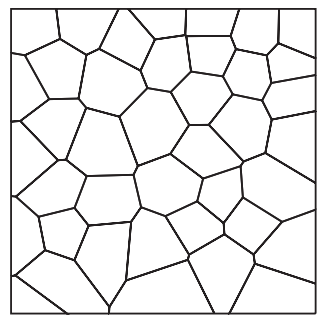

(c)

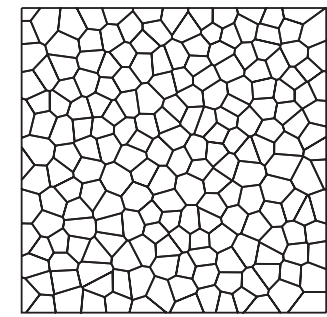

(d)

Figure 12. Patch test: (a) mesh $a$ (10 nodes); (b) mesh $b$ (22 nodes); (c) mesh $c$ (82 nodes); and (d) mesh $d$ (402 nodes).

Table I. Relative error in the $L^{2}$ norm for the patch test.

\begin{tabular}{|c|c|c|c|c|c|}
\hline \multirow[b]{2}{*}{ Meshes } & \multirow[b]{2}{*}{$\mathrm{nsp}$} & \multicolumn{4}{|c|}{$\frac{\left\|u-u^{h}\right\|_{L^{2}(\Omega)}}{\|u\|_{L^{2}(\Omega)}}$} \\
\hline & & $\begin{array}{l}\text { Wachspress } \\
\text { (Physical) }\end{array}$ & $\begin{array}{l}\text { Mean value } \\
\text { (Physical) }\end{array}$ & $\begin{array}{l}\text { Laplace } \\
\text { (Physical) }\end{array}$ & $\begin{array}{c}\text { Laplace } \\
\text { (Reference) }\end{array}$ \\
\hline \multirow{4}{*}{$\mathrm{a}$} & 3 & $4.3 \times 10^{-4}$ & $3.5 \times 10^{-4}$ & $4.0 \times 10^{-4}$ & $2.2 \times 10^{-5}$ \\
\hline & 6 & $3.5 \times 10^{-5}$ & $1.1 \times 10^{-4}$ & $5.1 \times 10^{-5}$ & $3.9 \times 10^{-7}$ \\
\hline & 13 & $2.6 \times 10^{-6}$ & $4.4 \times 10^{-5}$ & $1.3 \times 10^{-5}$ & $2.0 \times 10^{-8}$ \\
\hline & 25 & $6.8 \times 10^{-9}$ & $9.0 \times 10^{-6}$ & $1.1 \times 10^{-7}$ & $2.0 \times 10^{-11}$ \\
\hline \multirow{4}{*}{ b } & 3 & $2.6 \times 10^{-3}$ & $1.0 \times 10^{-3}$ & $1.4 \times 10^{-3}$ & $4.4 \times 10^{-4}$ \\
\hline & 6 & $2.6 \times 10^{-4}$ & $2.1 \times 10^{-4}$ & $2.9 \times 10^{-4}$ & $2.5 \times 10^{-5}$ \\
\hline & 13 & $2.5 \times 10^{-4}$ & $1.1 \times 10^{-4}$ & $1.1 \times 10^{-4}$ & $7.2 \times 10^{-7}$ \\
\hline & 25 & $4.7 \times 10^{-6}$ & $2.4 \times 10^{-5}$ & $4.5 \times 10^{-6}$ & $4.6 \times 10^{-9}$ \\
\hline \multirow{4}{*}{$\mathrm{c}$} & 3 & $1.7 \times 10^{-3}$ & $9.7 \times 10^{-4}$ & $1.4 \times 10^{-3}$ & $2.5 \times 10^{-4}$ \\
\hline & 6 & $3.5 \times 10^{-4}$ & $1.8 \times 10^{-4}$ & $2.6 \times 10^{-4}$ & $1.5 \times 10^{-5}$ \\
\hline & 13 & $1.0 \times 10^{-4}$ & $7.8 \times 10^{-5}$ & $8.0 \times 10^{-5}$ & $3.4 \times 10^{-7}$ \\
\hline & 25 & $6.5 \times 10^{-6}$ & $3.1 \times 10^{-5}$ & $6.7 \times 10^{-6}$ & $1.9 \times 10^{-9}$ \\
\hline \multirow{4}{*}{$\mathrm{d}$} & 3 & $9.2 \times 10^{-4}$ & $5.6 \times 10^{-4}$ & $7.2 \times 10^{-4}$ & $1.9 \times 10^{-4}$ \\
\hline & 6 & $2.5 \times 10^{-4}$ & $1.4 \times 10^{-4}$ & $1.9 \times 10^{-4}$ & $1.6 \times 10^{-5}$ \\
\hline & 13 & $1.1 \times 10^{-4}$ & $7.1 \times 10^{-5}$ & $8.8 \times 10^{-5}$ & $9.9 \times 10^{-7}$ \\
\hline & 25 & $2.4 \times 10^{-5}$ & $2.6 \times 10^{-5}$ & $2.5 \times 10^{-5}$ & $2.5 \times 10^{-9}$ \\
\hline
\end{tabular}

Numerical integration on the reference (canonical) element provide the most accurate results with the Laplace shape function; if this integration scheme is used for the mean value or Wachspress shape functions, there is marginal improvement in accuracy, and hence these results are not reported. Relative errors in the $L^{2}$ norm of $\mathcal{O}\left(10^{-6}\right)$ and $\mathcal{O}\left(10^{-9}\right)$ are realized with 13and 25-point quadrature schemes, respectively. This accuracy is significantly better than that attained in the natural element method [24] or in most of the other meshfree methods [18]. We attribute this to the conformity of the method, which was shown in Section 4.1, and the 
Table II. Relative error in the energy norm for the patch test.

\begin{tabular}{|c|c|c|c|c|c|}
\hline \multirow[b]{2}{*}{ Meshes } & \multirow[b]{2}{*}{ nsp } & \multicolumn{4}{|c|}{$\frac{\left\|u-u^{h}\right\|_{E(\Omega)}}{\|u\|_{E(\Omega)}}$} \\
\hline & & $\begin{array}{l}\text { Wachspress } \\
\text { (Physical) }\end{array}$ & $\begin{array}{c}\text { Mean value } \\
\text { (Physical) }\end{array}$ & $\begin{array}{l}\text { Laplace } \\
\text { (Physical) }\end{array}$ & $\begin{array}{c}\text { Laplace } \\
\text { (Reference) }\end{array}$ \\
\hline \multirow{4}{*}{$\mathrm{a}$} & 3 & $9.9 \times 10^{-4}$ & $5.3 \times 10^{-4}$ & $9.0 \times 10^{-4}$ & $5.8 \times 10^{-5}$ \\
\hline & 6 & $8.7 \times 10^{-5}$ & $1.7 \times 10^{-4}$ & $1.2 \times 10^{-4}$ & $1.0 \times 10^{-6}$ \\
\hline & 13 & $6.3 \times 10^{-6}$ & $6.7 \times 10^{-5}$ & $3.3 \times 10^{-5}$ & $5.3 \times 10^{-8}$ \\
\hline & 25 & $1.7 \times 10^{-8}$ & $1.6 \times 10^{-5}$ & $2.7 \times 10^{-7}$ & $5.3 \times 10^{-11}$ \\
\hline \multirow{4}{*}{ b } & 3 & $1.3 \times 10^{-2}$ & $4.6 \times 10^{-3}$ & $6.8 \times 10^{-3}$ & $2.3 \times 10^{-3}$ \\
\hline & 6 & $4.1 \times 10^{-3}$ & $8.9 \times 10^{-4}$ & $1.4 \times 10^{-3}$ & $1.3 \times 10^{-4}$ \\
\hline & 13 & $1.3 \times 10^{-3}$ & $4.9 \times 10^{-4}$ & $5.5 \times 10^{-4}$ & $3.4 \times 10^{-6}$ \\
\hline & 25 & $2.6 \times 10^{-5}$ & $1.1 \times 10^{-4}$ & $2.3 \times 10^{-5}$ & $2.4 \times 10^{-8}$ \\
\hline \multirow{4}{*}{$\mathrm{c}$} & 3 & $2.1 \times 10^{-2}$ & $1.0 \times 10^{-2}$ & $1.6 \times 10^{-2}$ & $1.7 \times 10^{-3}$ \\
\hline & 6 & $4.6 \times 10^{-3}$ & $1.9 \times 10^{-3}$ & $3.1 \times 10^{-3}$ & $9.4 \times 10^{-5}$ \\
\hline & 13 & $1.4 \times 10^{-3}$ & $8.3 \times 10^{-4}$ & $9.8 \times 10^{-4}$ & $2.9 \times 10^{-6}$ \\
\hline & 25 & $8.0 \times 10^{-5}$ & $3.3 \times 10^{-4}$ & $9.4 \times 10^{-5}$ & $1.5 \times 10^{-8}$ \\
\hline \multirow{4}{*}{$\mathrm{d}$} & 3 & $3.0 \times 10^{-2}$ & $1.8 \times 10^{-2}$ & $2.3 \times 10^{-2}$ & $3.1 \times 10^{-3}$ \\
\hline & 6 & $8.5 \times 10^{-3}$ & $4.7 \times 10^{-3}$ & $6.2 \times 10^{-3}$ & $2.6 \times 10^{-4}$ \\
\hline & 13 & $3.9 \times 10^{-3}$ & $2.4 \times 10^{-3}$ & $3.1 \times 10^{-3}$ & $1.4 \times 10^{-5}$ \\
\hline & 25 & $9.1 \times 10^{-4}$ & $9.4 \times 10^{-4}$ & $9.4 \times 10^{-4}$ & $5.3 \times 10^{-8}$ \\
\hline
\end{tabular}

fact that the support of the shape functions coincide with the domains used in the numerical integration of the bilinear form.

\subsection{Convergence study}

The accuracy and convergence of the methods are studied for the model Poisson problem given in Equation (22). We use $f\left(x_{1}, x_{2}\right)=2\left(1-x_{1}^{2}\right)+2\left(1-x_{2}^{2}\right)$, and hence the exact solution is: $u\left(x_{1}, x_{2}\right)=\left(1-x_{1}^{2}\right)\left(1-x_{2}^{2}\right)$. Two different types of meshes are used in the convergence study. In the first instance, to ensure uniform refinement, hexagonal meshes with edge length of $h$ in the bulk of the domain are used; nodal spacing differs only near the boundary where essential boundary conditions are imposed. Five meshes with predominantly hexagonal elements are considered with $74\left(h=1.597 \times 10^{-1}\right), 884\left(h=5.082 \times 10^{-2}\right), 1802(h=3.607 \times$ $\left.10^{-2}\right), 2740\left(h=2.942 \times 10^{-2}\right)$, and $9524\left(h=1.597 \times 10^{-2}\right)$ nodes. In the second case, we use quasi-regular meshes with a fairly uniform distribution of nodes within the bi-unit square. Four different meshes with 576, 864, 2123, and 5611 nodes are used. A sample mesh for each study is depicted in Figure 13. In Figure 14, the relative error in the $L^{2}$ and energy norms are plotted versus $\sqrt{M}$ ( $M$ is the number of nodes) on a log-log scale. The slope of the plots, $\lambda$, indicates the rate of convergence of the method. From Figure 14, we obtain $\lambda=2$ for the rate of convergence in $u$ and $\lambda=1$ for the convergence rate in the energy, which are consistent with the theoretical predictions for second-order elliptic PDEs with linearly complete approximations [17]. 


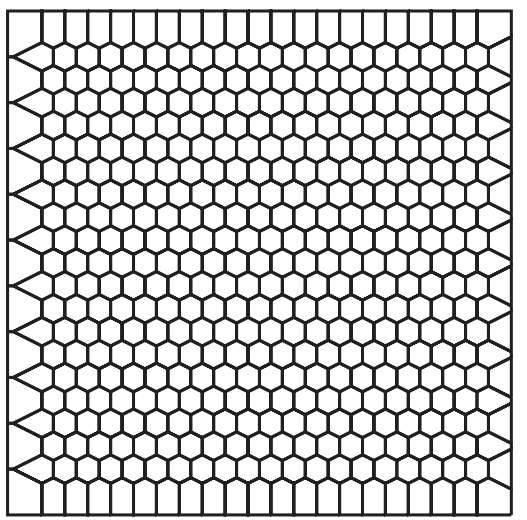

(a)

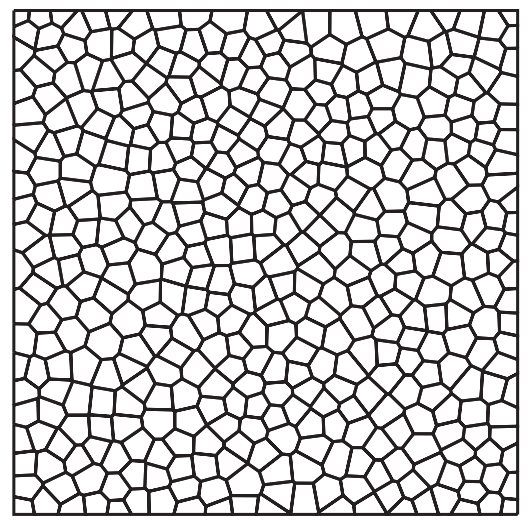

(b)

Figure 13. Sample meshes used in the convergence study: (a) hexagonal mesh (884 nodes); and (b) quasi-regular mesh (864 nodes).
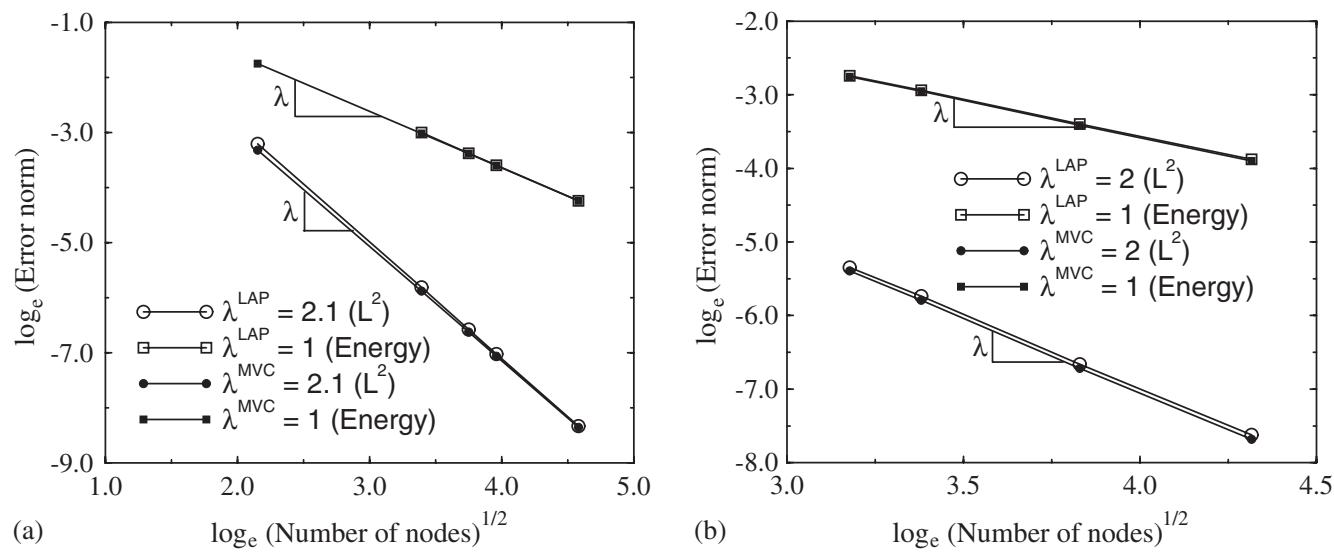

Figure 14. Rate of convergence in the $L^{2}(\Omega)$ and energy error norms: (a) hexagonal meshes; and (b) quasi-regular meshes.

\section{CONCLUDING REMARKS}

In this paper, we presented the construction and numerical implementation of three conforming polygonal finite element methods. The rational finite element basis function proposed by Wachspress [3], and revisited by Meyer and co-workers [42], was used as a point of reference. The mean value (barycentric) co-ordinate [43], which was recently introduced in computer graphics, was considered within a Galerkin procedure, and in addition, the construction of a conforming approximation using a natural neighbour (Laplace) interpolant [28] was proposed. In the spirit of three- and four-node finite elements, the Laplace shape functions were defined 
on a canonical (reference) element and an affine map was used to obtain the shape functions and their gradients on irregular polygons. The attractive properties of the Laplace interpolant (interpolation, linear completeness, and linear behaviour on the boundary of a convex polygon) permitted the direct imposition of essential boundary conditions as is done in classical finite element methods. We proved that the Laplace and Wachspress shape functions were identical on regular $n$-gons, and the conformity of the mean value and Laplace interpolants were also numerically verified.

Numerical results for the patch test and the accuracy and rate of convergence for a Poisson problem were presented. Patch test results using the Laplace interpolant were the most accurate, and the error in the $L^{2}(\Omega)$ norm of $\mathcal{O}\left(10^{-9}\right)$ was obtained when numerical integration was performed on the canonical element. The rate of convergence in the $L^{2}(\Omega)$ norm was found to be two, whereas that in the energy norm was unity. These results are consistent with theory for linearly complete interpolants such as those considered in this study. In conclusion, we are optimistic that the proposed finite element method can open-up new pathways in finite element research, and also lead to the wider adoption and advancement of the use of polygonal finite element methods in solid mechanics and computational materials science. For example, (i) Polygonal and polyhedral mesh generation algorithms; (ii) Adaptive computations on quadtree [49] and octree meshes; and (iii) Application of the polygonal FEM in material design and in the modelling of fracture phenomenon, are some of the potential topics for future research.

\section{ACKNOWLEDGEMENTS}

The authors are grateful for the research support of the National Science Foundation through contract CMS-0352654 to the University of California, Davis. We also thank Professor John Bolander for providing the code for the Voronoi mesh generator, which was used to generate the polygonal meshes considered in this study.

\section{REFERENCES}

1. Hughes TJR. The Finite Element Method. Prentice-Hall: Englewood Cliffs, NJ, 1987.

2. Bathe KJ. Finite Element Procedures. Prentice-Hall: Englewood Cliffs, NJ, 1996.

3. Wachspress EL. A Rational Finite Element Basis. Academic Press: New York, NY, 1975.

4. Gout JL. Rational Wachspress-type finite elements on regular hexagons. IMA Journal of Numerical Analysis 1985; 5:59-77.

5. Dahmen W, Dikshit HP, Ojha A. On Wachspress quadrilateral patches. Computer Aided Geometric Design 2000; 17:879-890.

6. Malsch EA, Dasgupta G. Shape functions for concave quadrilaterals. In Proceedings of the First MIT Conference on Fluid and Solid Mechanics, vol. 2, Bathe KJ (ed.). Amsterdam. Elsevier Press: The Netherlands, 2001; 1617-1622.

7. Dasgupta G. Interpolants within convex polygons: Wachspress' shape functions. Journal of Aerospace Engineering 2003; 16(1):1-8.

8. Dasgupta G. Integration within polygonal finite elements. Journal of Aerospace Engineering 2003; 16(1): 9-18.

9. Martin RB, Burr DB, Sharkey AN. Skeletal Tissue Mechanics. Springer-Verlag: New York, NY, U.S.A., 1998.

10. Cueto E, Calvo B, Doblaré M. Modelling three-dimensional piece-wise homogeneous domains using the $\alpha$-shape-based natural element method. International Journal for Numerical Methods in Engineering 2002; 54(6):871-897.

11. Peters JF, Heymsfield E. Application of the 2-D constant strain assumption to FEM elements consisting of an arbitrary number of nodes. International Journal for Numerical Methods in Engineering 2003; 40: $143-159$. 
12. Dohrmann CR, Key SW, Heinstein MW. A method for connecting dissimilar finite element meshes in two dimensions. International Journal for Numerical Methods in Engineering 2000; 48:655-678.

13. Diaz AR, Bénard A. Designing materials with prescribed elastic properties using polygonal cells. International Journal for Numerical Methods in Engineering 2003; 57(3):301-314.

14. Ghosh S, Moorthy S. Elastic-plastic analysis of arbitrary heterogeneous materials with the Voronoi cell finite-element method. Computer Methods in Applied Mechanics and Engineering 1995; 121(1-4):373-409.

15. Okabe A, Boots B, Sugihara K. Spatial Tessellations: Concepts and Applications of Voronoi Diagrams. John Wiley \& Sons: Chichester, England, 1992.

16. Christie I, Hall C. The maximum principle for bilinear elements. International Journal for Numerical Methods in Engineering 1984; 20:549-553.

17. Strang G, Fix G. An Analysis of the Finite Element Method. Prentice-Hall: Englewood Cliffs, NJ, 1973.

18. Belytschko T, Krongauz Y, Organ D, Fleming M, Krysl P. Meshless methods: An overview and recent developments. Computer Methods in Applied Mechanics and Engineering 1996; 139:3-47.

19. Li S, Liu WK. Meshfree and particle methods and their applications. Applied Mechanics Review 2002; 55(1):1-34.

20. Lancaster P, Salkauskas K. Surfaces generated by moving least squares methods. Mathematics of Computation 1981; 37:141-158.

21. Babuška I, Banerjee U, Osborn JE. Survey of meshless and generalized finite element methods: A unified approach. Acta Numerica 2003; 12:1-125.

22. Braun J, Sambridge M. A numerical method for solving partial differential equations on highly irregular evolving grids. Nature 1995; 376:655-660.

23. Sukumar N. The Natural Element Method in Solid Mechanics. Ph.D. Thesis, Theoretical and Applied Mechanics, Northwestern University, Evanston, IL, U.S.A., June 1998. URL: http://dilbert.engr. ucdavis. edu/ suku/nem/thesis.html.

24. Sukumar N, Moran B, Belytschko T. The natural element method in solid mechanics. International Journal for Numerical Methods in Engineering 1998; 43(5):839-887.

25. Sukumar N, Moran B, Semenov AYu, Belikov VV. Natural neighbor Galerkin methods. International Journal for Numerical Methods in Engineering 2001; 50(1):1-27.

26. Yoo JW, Moran B, Chen JS. Stabilized conforming nodal integration in the natural-element method. International Journal for Numerical Methods in Engineering 2004; 60(5):861-890.

27. Sibson R. A vector identity for the Dirichlet tessellation. Mathematical Proceedings of the Cambridge Philosophical Society 1980; 87:151-155.

28. Christ NH, Friedberg R, Lee TD. Weights of links and plaquettes in a random lattice. Nuclear Physics B 1982; 210(3):337-346.

29. Belikov VV, Ivanov VD, Kontorovich VK, Korytnik SA, Semenov AYu. The non-Sibsonian interpolation: A new method of interpolation of the values of a function on an arbitrary set of points. Computational Mathematics and Mathematical Physics 1997; 37(1):9-15.

30. Hiyoshi H, Sugihara K. Two generalizations of an interpolant based on Voronoi diagrams. International Journal of Shape Modeling 1999; 5(2):219-231.

31. Cueto E, Sukumar N, Calvo B, Martínez MA, Cegon̈ino J, Doblaré M. Overview and recent advances in natural neighbor Galerkin methods. Archives of Computational Methods in Engineering 2003; 10(4):307-384.

32. Sukumar N. Voronoi cell finite difference method for the diffusion operator on arbitrary unstructured grids. International Journal for Numerical Methods in Engineering 2003; 57(1):1-34.

33. Sukumar N, Bolander JE. Numerical computation of discrete differential operators on non-uniform grids. CMES: Computer Modeling in Engineering and Sciences 2003; 4(6):691-706.

34. Hiyoshi H, Sugihara K. Improved continuity of Voronoi-based interpolation over Delaunay spheres. Computational Geometry 2002; 22:167-183.

35. Idelsohn SR, Calvo N, Onate E. Polyhedralization of an arbitrary 3D point set. Computer Methods in Applied Mechanics and Engineering 2003; 192(22-24):2649-2667.

36. Idelsohn SR, Onate E, Calvo N, Del Pin F. Meshless finite element method. International Journal for Numerical Methods in Engineering 2003; 58(6):893-912.

37. Coxeter HSM. Introduction to Geometry. Wiley: New York, NY, 1961.

38. Warren J. Barycentric coordinates for convex polytopes. Advances in Computational Mathematics 1996; 6(1):97-108.

39. Rashid MM, Gullett PM. On a finite element method with variable element topology. Computer Methods in Applied Mechanics and Engineering 2000; 190(11-12):1509-1527. 
40. Bazeley GR, Cheung YK, Irons BM, Zienkiewicz OC. Triangular elements in plate bending. Conforming and non-conforming solutions. In Proceedings of the 1st Conference on Matrix Methods in Structural Mechanics, Wright-Patterson AFB, 1965.

41. Irons BM, Razzaque A. Experience with the patch test for convergence of finite elements. In The Mathematical Foundations of the Finite Element Method with Applications to Partial Differential Equations, Aziz AK (ed.). Academic Press: New York, 1972.

42. Meyer M, Lee H, Barr AH, Desbrun M. Generalized barycentric coordinates for irregular n-gons. Journal of Graphics Tools 2002; 7(1):13-22.

43. Floater MS. Mean value coordinates. Computer Aided Geometric Design 2003; 20(1):19-27.

44. Floater MS. One-to-one piecewise linear mappings over triangulations. Mathematics of Computations 2003; 72(242):685-696.

45. Abramowitz M, Stegun IA. Handbook of Mathematical Functions with Formulas, Graphs, and Mathematical Tables (10th edn). Applied Mathematics Series • 55. National Bureau of Standards: Washington DC, 1972.

46. Wandzura S, Xiao H. Symmetric quadrature rules on a triangle. Computers and Mathematics with Applications 2003; 45:1829-1840.

47. Xiao H. Private communication. 2003.

48. Sukumar N, Prévost J-H. Modeling quasi-static crack growth with the extended finite element method. Part I: Computer implementation. International Journal of Solids and Structures 2003; 40(26):7513-7537.

49. Tabarraei A, Sukumar N. Adaptive computations on conforming quadtree meshes. Finite Elements in Analysis and Design (Special Issue on Papers Presented at the Robert J. Melosh Finite Element Medal Competition), to appear 2005. 